\title{
Neoantigens and their potential applications in tumor immunotherapy (Review)
}

\author{
XIANZHU FANG $^{1 *}$, ZHILIANG GUO $^{2 *}$, JINQING LIANG $^{1}$, JIAO WEN $^{1}$, \\ YUANYUAN LIU ${ }^{1}$, XIUMEI GUAN ${ }^{1^{*}}$ and HONG LI ${ }^{*}$ \\ ${ }^{1}$ Department of Biochemistry and Molecular Biology, School of Basic Medical Sciences, Weifang Medical University, \\ Weifang, Shandong 261053; ${ }^{2}$ Department of Orthopedic, The 80th Group Army Hospital of \\ Chinese People's Liberation Army, Weifang, Shandong 261021, P.R. China
}

Received September 13, 2021; Accepted January 4, 2022

DOI: $10.3892 / \mathrm{ol} .2022 .13208$

\begin{abstract}
The incidence of malignant tumors is increasing, the majority of which are associated with high morbidity and mortality rates worldwide. The traditional treatment method for malignant tumors is surgery, coupled with radiotherapy or chemotherapy. However, these therapeutic strategies are frequently accompanied with adverse side effects. Over recent decades, tumor immunotherapy shown promise in demonstrating notable efficacy for the treatment of cancer. With the development of sequencing technology and bioinformatics algorithms, neoantigens have become compelling targets for cancer immunotherapy due to high levels of immunogenicity. In addition, neoantigen-based vaccines have demonstrated potential for cancer therapy, primarily by augmenting T-cell responses. Neoantigens have also been shown to be effective in immune checkpoint blockade therapy. Therefore, neoantigens may serve to be predictive biomarkers and synergistic treatment targets in cancer immunotherapy. The aim of the present review was to provide an overview of the recent progress in the classification, screening and clinical application of neoantigens for cancer therapy.
\end{abstract}

\section{Contents}

1. Introduction

2. Tumor immunology and tumor immunotherapy

3. Classification and screening of neoantigens

4. Neoantigen-based tumor vaccines

Correspondence to: Dr Hong Li, Department of Biochemistry and Molecular Biology, School of Basic Medical Sciences, Weifang Medical University, 7166 West Baotong Road, Weifang, Shandong 261053, P.R. China

E-mail:1h@wfmc.edu.cn

${ }^{*}$ Contributed equally

Key words: neoantigen, immunotherapy, tumor vaccine, immune checkpoint blockade
5. Application of neoantigens in immune checkpoint blockade therapy

6. Adoptive cellular therapies (ACT) targeting neoantigens

7. Outlook

\section{Introduction}

Harmful stimuli, including ultraviolet radiation, ionizing radiation and carcinogens, can result in single-nucleotide mutations, insertions or deletions, gene fusion, frameshift mutations, structural mutations or integration and clonal expansion of the tumor-associated virus genome within the human genome $(1,2)$. In particular, these genetic alterations can also cause somatic cell mutagenesis (3-6). Over the past number of decades, immunotherapy has demonstrated great potential for the treatment of cancer. This is because tumor cells produce mutant proteins that can be recognized by the immune system as antigens, which trigger cellular and humoral immune responses downstream. Some non-synonymous mutations can give rise to mutated, non-self peptides that can be presented by human (HLA) molecules and elicit T-cell responses, which are known as neoantigens (7). Since leukocyte antigen neoantigens are not affected by thymus selection or central tolerance, $\mathrm{T}$ cells exhibiting high-avidity likely exist (8). Therefore, immunotherapy of malignant tumors by targeting these non-synonymous mutant proteins is a research field that is garnering significant interest (9).

\section{Tumor immunology and tumor immunotherapy}

Chen and Mellman (10) previously proposed a cancer-immune cycle theory to explain a potentially key role of cancer immunotherapy in the clinical management of cancer (Fig. 1). Specifically, the antigens produced by necrotic tumor cells can be captured by dendritic cells (DCs) (10). In the DCs, antigen polypeptides are digested, fragmented and transported into the endoplasmic reticulum (ER) (10). In the ER, the peptides encounter ER aminopeptidase related to antigen processing and major histocompatibility complex (MHC) class I molecules to form the peptide-loading complex (10). After this MHC-I complex reaches a certain stability threshold, it will leave the 
ER and reach the cell surface, functioning as a potential ligand for the T-cell receptor (TCR) on CD8 ${ }^{+}$T cells (Fig. 2) $(11,12$ ).

Previous studies have demonstrated that antigens produced by necrotic tumor cells also have the ability to bind to MHC-II molecules, which are associated with the functions of $\mathrm{CD}^{+}$ $\mathrm{T}$ cells $(13,14)$. Activated effector T cells recognize and attack cancer cells in the tumor bed. Additional tumor-associated antigens (TAAs) are released by dead cancer cells, which increases the breadth and depth of the cancer-immune cycle (10).

Techniques for tumor immunotherapy have developed over the past decade, such that notable antitumor activities have been reported for the treatment of numerous solid tumors, including melanoma, non-small cell lung cancer (NSCLC), kidney cancer, prostate cancer and glioblastoma (15-17). Tumor immunotherapy includes immune enhancement therapy, tumor vaccines, immune checkpoint blockade therapy and adoptive cellular therapy (ACT). Immunotherapeutic agents, including immune checkpoint blockers targeting the programmed cell death (PD)-1/PD-ligand (L)1 signaling pathway, have been approved by the US Food and Drug Administration (FDA) for clinical application (18). In addition, results of a previous study demonstrated that the neoantigen burden in tumor tissues is directly and positively associated with the tumor mutational burden (TMB). TMB is an indicator of the tumor mutation quantity, which translates into the structures of neoantigens and is presented to T cells by MHC proteins (19). In particular, melanoma has a high mutation rate, meaning that PD-1 antibodies are more likely to mediate beneficial effects. Furthermore, approved immune checkpoint inhibitors mainly target tumors with high TMB and neoantigen loads, including melanoma, urothelial cancer and NSCLC (20). For solid tumors with low mutation loads, it would be more appropriate to apply immunotherapy based on neoantigens (tumor vaccines and adoptive cell therapies), as neoantigens have high tumor specificity, without being affected by thymus selection and the lack of central tolerance $(8,21)$.

\section{Classification and screening of neoantigens}

Based on previous clinical and tumor immunology data, tumor cell epitopes can be classified into two categories $(8,22)$. The first category is TAAs, which are formed by nonmutant proteins and are not unique to tumor cells. This type of antigen also exists in non-cancerous cells but is instead aberrantly expressed during carcinogenesis. The second category includes peptides that exist only in tumor cells or a specific tumor cell type, known as tumor-specific antigens (TSAs) or neoantigens (8).

Classification of neoantigens. Neoantigens are antigens arising from somatic mutations that generate these mutant peptides, which are processed and presented by MHC on the cell surface. Neoantigens exhibiting potent immunogenicity are not normally present in healthy cells or tissues and can activate the immune system to eliminate tumor cells (23). Therefore, neoantigens are attractive targets for designing precision immunotherapeutic stratgies, such as antibodies, vaccines and cellular therapeutics. At present, neoantigens are classified into the following two types: Private and public neoantigens. Private neoantigens are mutated antigens that are unique to most neoantigens and typically differ among patients. Therefore, therapeutic strategies based on private neoantigens are designed to the specification of each patient and are also named personalized therapy (24). Previous clinical data demonstrated that tumor-infiltrating lymphocytes (TILs) from patients with gastrointestinal cancer can recognize neoantigens expressed by tumor cells due to somatic mutations, where the majority of the neoantigen determinants were unique and not shared among patients (25). By contrast, public neoantigens refer to mutated antigens that are shared and conserved among patients with cancer. Immunotherapies targeting public neoantigens are applicable to groups of patients with analogous genetic alterations (26). Neoantigens can induce immune responses with high specificity to cancer cells because of their underlying mutations, whilst exerting minimal toxicity to non-cancerous cells. Therefore, screening for novel tumor neoantigens may serve to be a useful strategy in cancer immunotherapy.

Screening for tumor neoantigens. A number of strategies have been devised to screen for candidate neoantigens, such as whole-exome sequencing (WES), computer algorithm and immunological effects evaluation (Fig. 3). With the development of sequencing technology, mutations can be screened using WES (27). If such mutant proteins are expressed highly in tumor cells, they exhibit the potential to be recognized as neoantigens (28). Subsequently, a computer algorithm can be used to predict the affinity of neoantigen peptides of interest to HLA-1 molecules. Peptides with increased predicted levels of immunogenicity may be selected to be neoantigen candidates. Neoantigen-specific $\mathrm{T}$ cells are thereby isolated from the tumor cell infiltration area or the peripheral blood samples of patients, expanded to $\mathrm{T}$ cells in vitro and then reinfused back into the body. Subsequently, the immunological efficacy of the candidate neoantigens can be assessed $(27,28)$. Previous reports from Chen et al (29) demonstrated that a large proportion of the immunogenic neo-epitopes were recognized by autologous $\mathrm{T}$ cells, rendering this a viable pipeline for neoantigen identification.

However, a number of limitations must be considered. Algorithms that are currently used for predicting neoantigens are limited by binding affinity data in vitro and computational constraints, resulting in a high false discovery rate (29). To circumvent this, Hao et al (30) proposed a deep convolutional neural network, named the antigen presentation prediction model (APPM), for predicting antigen presentation. The positive predictive value of APPM, combined with the immune epitope database, can optimize the accuracy further for predicting neoantigens (28). In addition, currently applied methods used for screening neoantigens are relevant to specific HLA alleles. Bulik-Sullivan et al (31) previously examined a large HLA peptide and genomic dataset from various human tumors to create a computational model named EDGE, which increased the positive predictive value of HLA antigen prediction by $\leq$ nine-fold.

\section{Neoantigen-based tumor vaccines}

At present, the most promising method in cancer immunotherapy is the development of therapeutic tumor vaccine based 



$\mathrm{CD}^{+} \mathrm{T}$ cell and DC cell interaction. CTLA4 and PD-L1 inhibit $T$ cell activation

\section{The Cancer-Immunity Cycle}

Antigens uptake by dendritic cells. This process occurs mainly in the endoplasmic reticulum. Key mediators include immune cell factors, proinflammatory cytokines, endogenous adjuvants, gut microbiome products.

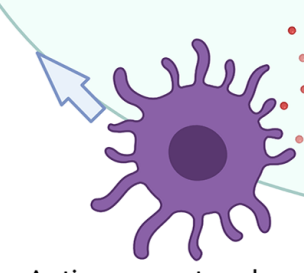

Antigens capture by dendritic cell

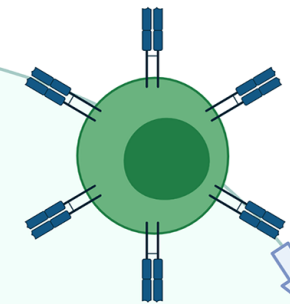

Trafficking of T cells to cancers

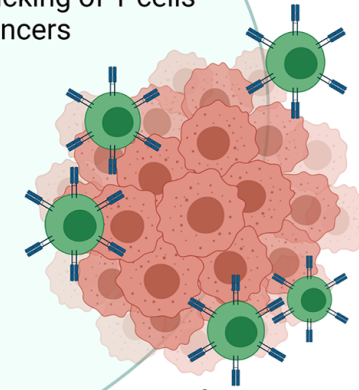

Generation of immunity to cancer is a cyclic process, where activators or inhibitors can accumulate.

Figure 1. Stages of cancer-immunity cycle. Necrotic tumor cell antigens are released and captured by DCs. DCs present the captured antigens to T cells, resulting in the activation of effector $\mathrm{T}$ cells. The activated effector $\mathrm{T}$ cells then recognize and bind to cancer cells. Tumor cells killed by $\mathrm{T}$ cells in turn release antigens that enter the immune cycle again to amplify the response in subsequent revolutions of the cycle. DCs, Dendritic cells; CTLA-4, cytotoxic T lymphocyte protein 4; PD-L1, programmed-death ligand 1.



Figure 2. MHC-I antigen complex formation. After DCs ingest antigen precursors, the polypeptides are fragmented and transported into the ER for further editing. The processed peptides then encounter MHC-I molecules within the PLC as well as ERAAP. When the MHC-I complex reaches a certain stability threshold, it leaves the ER and reaches the cell surface for antigen presentation. MHC, major histocompatibility complex; DCs, dendritic cells; ER, endoplasmic reticulum; PLC, peptide-loading complex; ERAAP, endoplasmic reticulum aminopeptidase related to antigen processing. 



Figure 3. Schematic illustration of the neoantigen screening and clinical application workflow. WES is typically performed on tumor and normal DNA to identify tumor-specific mutations. Next, a computer algorithm is used to predict the affinity of neoantigen peptides to HLA-1 molecules. The immunological effects of candidate neoantigens were then evaluated as to whether they could be recognized by autologous T cells. Finally, the immunotherapies, including neoantigen vaccines, ACT based on neoantigens or combination therapies with checkpoint inhibitors are applied in the clinic. HLA, leukocyte antigen; WES, whole-exome sequencing; ACT, adoptive cellular immunotherapy.

on neoantigens. The benefits of this vaccine type are less tolerance compared with other traditional therapeutic drugs such as Tarceva, Gleevec and Herceptin, which enables it to activate the patient's own immune system to induce a sustained antitumor response (32-34). Despite numerous efforts to develop cancer vaccines, their conversion into efficacious clinical therapy have been challenging, with an objective clinical response rate of only $>7 \%$ and an overall rate of clinical benefit of only $220 \%$ (35). To achieve the full potential of cancer vaccines, personalized neoantigen vaccines have been introduced (23). Personalized neoantigen vaccines include DC-, DNA-, RNAand synthetic peptide-based vaccines, some of which are currently undergoing clinical trials (Table I).

Tumor lytic products. Tumor lytic products are some of the earliest immune vaccines to be applied for tumor therapy (36). Tumor cells are typically obtained during surgery and subsequently digested either by irradiation or tumor cell lysis. Complete tumor lysate contains all potential antigens (TAAs and TSAs), including neoantigens. Several clinical trials have begun with using tumor lytic products. Chiang et al (37) previously used hypochlorite to oxidize the cleavage products to enhance antigenicity, which improved treatment efficacy by DCs. Bencherif et al (38) demonstrated a cryogen-based whole tumor cell vaccine containing DC-activating factors, such as granulocyte-macrophage colony-stimulating factor, which can be used for injection. This vaccine has been demonstrated to be capable of regressing melanoma in mice (33). However, despite intensive research efforts into developing autologous tumor cells, a myriad of problems remain to be solved, including the maintenance of large-scale tumor cell culture, control of vaccine quality and standardization of vaccine production.

Protein/peptide vaccines. Protein/peptide vaccines have been extensively studied in cancer therapy trials due to their safety, cost effectiveness and ease of storage. Nevertheless, due to the high variety of unique peptide epitopes, tendency to degrade easily and low molecular weights, protein/peptide vaccines exhibit two main limitations: Low immunogenicity and $\mathrm{MHC}$ restriction. Previous studies have demonstrated that the addition of an immune adjuvant to the peptide vaccine is essential for inducing an effective immune response $(39,40)$. Traditional adjuvants, such as Freund's, bacterial and cytokine adjuvants (41), have all been used to activate the body's immune system and maintain the structure of the antigen. In addition, advances in nanotechnology have created opportunities for the development of novel types of adjuvants. For example, 5-100 $\mathrm{nm}$ nanovaccines (IL-2 and a lymphoma-specific antigen into liposomal particles) were found to be retained in lymphoid tissues with advanced-stage follicular lymphoma for a prolonged period, so that they can easily recognized and presented by immune cells in the lymphatic system (42).

For neoantigens, preparation of a peptide vaccine is key due to the high levels of immunogenicity (43). It has previously been suggested that new types of adjuvants coupled with neoantigen peptides, including charge-modified peptide-toll-like receptor (TLR)-7/8a conjugates assembled into nanoparticles, can significantly improve the cytotoxicity 
Table I. Selected clinical trials based on personalized neoantigen vaccines.

\begin{tabular}{|c|c|c|c|}
\hline $\begin{array}{l}\text { ClinicalTrials. } \\
\text { gov identifier }\end{array}$ & Treatment target & Type of vaccine & Composition \\
\hline NCT01970358 & Melanoma & Peptide vaccine & $\begin{array}{l}\text { In total, } 20 \text { neoantigens per patient, admixed with the } \\
\text { Toll-like receptor } 3 \text { agonist poly-ICLC }\end{array}$ \\
\hline NCT02897765 & $\begin{array}{l}\text { Melanoma, non-small cell } \\
\text { carcinoma, bladder cancer }\end{array}$ & Peptide vaccine & $\begin{array}{l}\text { Composed of } 20 \text { unique peptides, ranging in length } \\
\text { from } 14 \text { to } 35 \text { amino acids }\end{array}$ \\
\hline NCT02287428 & Glioblastoma & Peptide vaccine & $\begin{array}{l}\text { In total, } 20 \text { neoantigens per patient, admixed with the } \\
\text { poly-ICLC }\end{array}$ \\
\hline NCT01846143 & Melanoma & Peptide vaccine & Phosphorylated peptide \\
\hline NCT02960230 & Diffuse midline glioma & Peptide vaccine & $\begin{array}{l}\text { Synthetic } \mathrm{H} 3.3 \mathrm{~K} 27 \mathrm{M}_{26-35} \text { peptide, helper tetanus } \\
\text { toxoid peptide and poly-ICLC }\end{array}$ \\
\hline NCT01461148 & $\begin{array}{l}\text { Mismatch Repair Deficient } \\
\text { Cancers (MMR-deficient } \\
\text { colorectal cancer) }\end{array}$ & Peptide vaccine & Between 13 and 30 amino acids \\
\hline NCT03480152 & Gastrointestinal cancer & mRNA vaccine & $\begin{array}{l}\text { mRNA skeleton composition encoding up to } \\
20 \text { different antigens }\end{array}$ \\
\hline NCT01209871 & Plasma cell lymphoma & DNA vaccine & $\begin{array}{l}\text { Fusion of antigen with sequence encoding chemokine } \\
\text { (Macrophage Inflammatory Protein- } 3 \alpha \text { ) }\end{array}$ \\
\hline NCT02163057 & $\begin{array}{l}\text { Squamous cell carcinoma } \\
\text { of the head and neck }\end{array}$ & DNA vaccine & $\begin{array}{l}\text { Targeting human papilloma virus } 16 / 18 \mathrm{E} 6 / \mathrm{E} 7 \\
\text { encoding plasmid and IL-12 as adjuvant }\end{array}$ \\
\hline
\end{tabular}

Poly-ICLC, polyinosinic and polycytidylic acid, stabilized with poly-1-lysine and carboxymethylcellulose.

of $\mathrm{CD}^{+} \mathrm{T}$ cells (44). Ni et al (45) previously prepared a bi-adjuvant neoantigen nanovaccine (banNV), containing a peptide neoantigen [ADP-dependent glucokinase (Adpgk)] along with two other adjuvants, namely the TLR 7/8 agonist R848 and TLR9 agonist CpG, for colorectal cancer immunotherapy in mice. Results from this previous study revealed a highly potent immunogenic effect of this banNV coupled with reduced acute systemic toxicity, suggesting that banNVs can serve as a potential therapeutic neoantigen vaccine for the treatment of cancer (38). A variety of novel technologies are currently under development with aims of faciliatating neoantigen-specific T-cell activation (46-48).

Neoantigens obtained by screening a single peptide epitope exhibits weak immunogenicity, short half-lives and high HLA restriction, such that patients typically mount an ineffective immune response following vaccination. Therefore, research efforts are currently focusing on the development of a multitude of personalized vaccines containing a variety of epitopes to enhance the antitumor response (49). After obtaining the potential private and public neoantigens, multiplex vaccines containing 2-5 neoantigens in the form of long synthetic peptides are developed (50). A personalized long peptide neoantigen vaccine containing 20 neoepitopes has been previously synthesized and injected into patients with phase Ib glioblastoma (41). The results demonstrated that neoantigen-specific $\mathrm{CD}^{+}$and $\mathrm{CD}^{+}{ }^{+} \mathrm{T}$ cells were able to infiltrate into the tumor (51). In addition, Zeng et al (52) previously reported a case of personalized neoantigen immunotherapy for renal collecting duct carcinoma (CDC). According to the patient's specific mutations, 13 neoantigens were screened and identified, following which the corresponding long peptide neoantigen vaccines were prepared (42). A total of 3 months later, biopsy samples collected from the CDC sites exhibited a lower mutant allele frequency corresponding to $92 \%$ of the neoantigens, suggesting that tumor cells harboring these neoantigens were effectively eliminated (42). Nevertheless, vaccines developed based on personalized neoantigens require a prolonged development period, which may delay the treatment of cancer (53). Therefore, vaccines designed based on public neoantigens may be the novel therapeutic agent with the highest potential for the treatment of cancer. A synthetic long peptide for isocitrate dehydrogenase was previously used to design a neoantigen vaccine using public neoantigens, which yielded promising results regarding the survival of patients with late-stage melanoma (54).

Autologous DC vaccines. DC-based tumor vaccines have revealed a high potential for both preclinical and clinical applications $(55,56)$. Since they are highly effective antigen-presenting cells (APCs), DCs serve an important role in the regulation of both innate and adaptive immune responses, in addition to having a unique ability to activate effector and memory $\mathrm{T}$ cells. DC vaccines loaded with antigens have been demonstrated to induce more potent immune responses compared with vaccines composed of only antigens and adjuvants (57-59). For example, the objective response rate of patients with metastatic melanoma treated with an antigen-adjuvanted vaccine was only $2.6 \%$, whilst that of metastatic melanoma treated with a DC vaccine was $9.5 \%$ (35). Therefore, neoantigen-based DC vaccines hold high potential for cancer therapy. In addition, Carreno et al (59) previously reported that DC vaccines loaded with neoantigens can trigger T-cell-specific responses, which enhanced the immune response in three patients 
with melanoma. In particular, two patients remained stable whereas one patient exhibited no adverse effects or cancer recurrence (44). In another study, Zhang et al (57) found that the neoantigen-pulsed DC vaccine was superior to the neoantigen peptide-adjuvant immune vaccine in activating the immune response and inhibiting murine lung carcinoma growth and spread. In addition, it was previously demonstrated that plasma cell-like DCs are also potent antitumor inducers. Plasma cell-like DCs expand the effects of neoantigens and increase the number of specific $\mathrm{CD}^{+} \mathrm{T}$ cells by presenting neoantigen peptides from melanoma (60).

Nucleic acid (DNA or mRNA) vaccines. Nucleic acid vaccines are anticipated to replace traditional vaccines in the near future due to their unique benefits. Specifically, nucleic acid vaccines are non-infectious, such that RNA vaccines cannot integrate into host cell genome, eliminating the possibility of insertion mutation (61). Additionally, nucleic acids can be quickly absorbed and expressed throughout the body with high levels of efficiency $(24,62)$. Nucleic acid vaccines can also be used to exploit the strong immunogenicity of neoantigens to reverse immune tolerance, turning 'cold' tumors into 'hot' tumors. Importantly, these types of vaccines can be rapidly developed in a cost-effective manner (63). At present, nucleic acid cancer vaccines targeting neoantigen s have been investigated in various clinical trials $(64,65)$. However, further investigation into the coding regions of the nucleic acids in the vaccines is required to improve the levels of immunogenicity. Tondini et al (66) previously designed a circular DNA vaccine that used a plasmid to express the three neoantigenic determinants (dolichyl-phosphate $\mathrm{N}$-acetylglucosaminephosphotransferase 1, RalBP1-associated Eps domain-containing 1 and Adpgk) before evaluating its efficacy in mice. The results obtained revealed that this polymer DNA vaccine induced prophylactic protection against the B16 melanoma expressing ovalbumin (49). Furthermore, Li et al (67) identified a novel $\mathrm{CpG}$ oligodeoxynucleotide for promoting the immune response to inhibit melanoma tumor growth effectively. Specifically, $\mathrm{CpG}$ combined with mRNA cancer vaccines exhibited improved antitumor efficacy (50). Overall, these aforementioned findings provide a novel theoretical basis for the development of DNA or mRNA vaccines to further emphasize the importance of immunotherapy strategy development $(66,68)$.

\section{Application of neoantigens in immune checkpoint blockade therapy}

Numerous types of regulatory signals that can negatively regulate the tumor-killing ability of T cells are named immune checkpoints. The therapeutic field designed to suppress these associated signaling pathways leading to T-cell exhaustion is named immune checkpoint blockade therapy. Immune checkpoint inhibitors can continuously enhance the immune function of T cells in cancer (69). Previous studies have reported that PD-1 is a key signaling molecule in tumor immune evasion, which exerts immunosuppressive effects by binding to PD-L1 to inhibit T-cell proliferation and activation $(69,70)$. In addition, cytotoxic T lymphocyte protein 4 (CTLA-4) has been shown to block T-cell activation by binding to CD80 or CD86 on APCs (71). CTLA-4 and PD-1/PD-L1 mono-antibodies are the most extensively used immune checkpoint blockers for cancer immunotherapy. Therefore, monoclonal antibodies have been designed to target these types of immune checkpoint molecules (CTLA-4 and PD-1/PD-L1) to eliminate immunosuppression, thereby restoring the antitumor immune response (72-76).

At present, FDA-approved immune checkpoint inhibitors include the following antibodies $(18,77)$ : i) In total, three anti-PD-1 antibodies, including pembrolizumab (Keytruda), nivolumab (Opdivo) and cemiplimab (Libtayo); ii) three anti-PD-L1 antibodies, including atezolizumab (Tecentriq), durvalumab (Imfinzi) and avelumab (Bavencio); and iii) an anti-CTLA-4 antibody, namely ipilimumab (Bristol-Myers Squibb). Antibodies targeting T-cell immune checkpoint receptors PD-1/PD-L1 have demonstrated notable efficacy against melanoma, NSCLC and glioblastoma (78-80). However, the sole use of immune checkpoint inhibitors confers limited effects on improving immune system functions and is exceptionally susceptible to drug resistance (20). Therefore, an effective strategy may be the combination of immune checkpoint blockers with immunotherapy based on neoantigens. The combination of neoantigen vaccines and immune checkpoint blockade therapy may enhance the ability of the immune system to recognize low-immunogenic molecules and shared TAAs by mimicking antigen epitope transmission and blocking the immune escape-associated pathway. The specific peptides produced by cancer cells bind to HLA molecules with high efficiency and are presented to $\mathrm{CD}^{+}$and $\mathrm{CD} 4^{+} \mathrm{T}$ cells by APCs, thereby inhibiting autoimmunity and maximizing the therapeutic effect of neoantigens (81). Liu et al (82) previously demonstrated that the efficacy of the combination of anti-PD-L1 antibody and a neoantigen vaccine was superior to that of anti-PD-L1 alone in an aggressive orthotopic murine glioblastoma model. Similarly, Duraiswamy et al (83) revealed that the efficacy of PD-1 and CTLA-4 dual-blockade combined with the neoantigen vaccine in suppressing CT26 colon carcinoma and ID8-VEGF ovarian carcinoma was mediated by restoring T-cell functions. These studies suggest that the combined therapy of neoantigen vaccines and immune checkpoint inhibitors holds great potential for the treatment of cancer.

\section{Adoptive cellular therapies (ACT) targeting neoantigens}

ACT was previously used to isolate immune cells, such as DCs, lymphokine-activated killer cells, TILs and cytokine-induced killer cells from patients for subsequent amplification in vitro prior to re-infusion (84). TCR is a T-cell-specific receptor that participates in antigen recognition by naturally-occurring $\mathrm{T}$ cells. Due to its unique structure and function, TCR only recognizes peptides bound to major MHC molecules (85). Follow-up immunotherapy following the in vitro amplification of TILs is a widely practiced treatment method (86). Tumor antigen-specific $\mathrm{T}$ cells can recognize antigenic epitopes on the surface of tumor cells and kill them. This has been frequently exploited for treating patients who did not respond well to immune checkpoint inhibitor therapy or surgery (21). ACT with TILs has been demonstrated to confer high levels of therapeutic efficacy in metastatic melanoma (74). In 10 patients with melanoma who were not previously treated with TIL infusion, they exhibited an overall response rate of 50\% (87). In addition, neoantigen-specific $\mathrm{T}$ cells were detected in the 
tumor-infiltrating $\mathrm{T}$ cells of three patients. In total, six of the nine detected neoantigens were found to increase the response of specific $\mathrm{T}$ lymphocytes in the peripheral blood after the infusion of TILs (88). TIL-based adoptive T-cell therapies targeting neoantigens have demonstrated potential in patients with metastatic breast cancer (89). This highlight a basis for the development of novel personalized ACT against cancer.

Specific $\mathrm{T}$ lymphocytes have been screened in the tumor-infiltrating area for amplification and reinfusion. Tran et al (90) identified a GTPase KRAS G12D-targeting mutation (KRAS treatment gene, codon 12 mutation) in metastatic colorectal cancer. Neoantigen (KRAS G12D)-specific cell therapy resulted in the significant regression of the cancer. Sun et al (91) created an RNA mutanome vaccine based on neoantigens, which activated neoantigen-reactive T (NRT) cells. Following the adoptive transfer of these NRT cells, they exerted a significant antitumor effect in mouse lung cancer (78). These results suggest that adoptive NRT cell therapy is a feasible and effective therapeutic approach for lung cancer.

It should be emphasized that the amplification of $\mathrm{T}$ cells from bodily fluids or tissues requires a complex procedure. Notably, it is difficult to obtain high-affinity $\mathrm{TCR}^{+} \mathrm{T}$ cells, where $\mathrm{T}$ cells amplified in vitro cannot survive in the recipient for a prolonged period of time following infusion. In addition, different types of antigens exhibit individual variations, even in tumors within the same tissue type (92). Therefore, it is difficult to share neoantigens among patients.

\section{Outlook}

Cancer immunotherapy has emerged as a novel strategy for treating malignant tumors. Specifically, immune responses targeting designed neoantigens has attracted considerable attraction according to findings from numerous clinical trials. Therefore, screening for novel neoantigens has become a key focus in the field of immunotherapy. With the rapid and continuous development of sequencing technology and bioinformatics algorithms, tumor mutation sites have been efficiently and accurately examined to accelerate this process. These neoantigens identified have been used as vaccines to stimulate the immune system and generate an antitumor response in patients with cancer.

However, a significant number of limitations remain that must be addressed prior to the broader application of neoantigen-targeting immunotherapies. During the development and progression of tumors, numerous neoantigens with high levels of diversity are produced, which limits the option for developing a standardized model. Furthermore, previous studies have reported that only a small fraction of non-synonymous mutations identified by tumor WES are immunogenic $(93,94)$. Therefore, screening for specific neoantigens associated with specific tumors is critical. Cancers treated using personalized immunotherapies, such as ACT or vaccinations, may also generate a potently immunosuppressive local environment to prevent the activation of neoantigen-specific $\mathrm{T}$ cells (95). Rational strategies are therefore required to identify candidate neoantigens and evaluate their immunogenicity. Further limitations include the loss of neoantigens with heterogeneous expression profiles inside the treated tumor, which may result in the selection of subclones devoid of the target neoantigen (76).
In conclusion, the emergence of novel therapies, including neoantigen vaccines and ACT based on neoantigens, is expected to revolutionize the treatment of cancer based on precision medicine. The use of neoantigen vaccines have demonstrated encouraging outcomes and are more ideally suited for combination therapies, including those with checkpoint inhibitors, surgery, radiation therapy and chemotherapy. In addition, neoantigen-based therapeutic strategies hold potential for the treatment of cancer, such that an increase in the spectra of human malignancies that can respond to cancer immunotherapy will be developed.

\section{Acknowledgements}

Not applicable.

\section{Funding}

The present review was supported by the National Natural Science Foundation of China (grant no. 81870237), the Foundation of Health Commission of Weifang (grant no. wfwsjk_2019_025), the Research Project of Shandong Provincial Education Department (grant no. J14LK12) and the Weifang Medical University Doctoral Startup Fund.

\section{Availability of data and materials}

Not applicable.

\section{Authors' contributions}

$\mathrm{XF}, \mathrm{ZG}$ and JL wrote the manuscript. JW drafted the figure and table and revised the manuscript. XG and HL reviewed and edited the manuscript. ZG and YL contributed to the conception and design of the article and acquired funding. Data authentication is not applicable. All authors have read and approved the final manuscript.

\section{Ethics approval and consent to participate}

Not applicable.

\section{Patient consent for publication}

Not applicable.

\section{Competing interests}

The authors declare that they have no competing interests.

\section{References}

1. Tomasetti $\mathrm{C}$ and Vogelstein B: Cancer etiology. Variation in cancer risk among tissues can be explained by the number of stem cell divisions. Science 347: 78-81, 2015.

2. Wu S, Powers S, Zhu W and Hannun YA: Substantial contribution of extrinsic risk factors to cancer development. Nature 529: 43-47, 2015.

3. Compagni A and Christofori G: Recent advances in research on multistage tumorigenesis. Br J Cancer 83: 1-5, 2000.

4. Paul S and Régulier E: Molecular basis of oncogenesis. Ann Biol Clin (Paris) 59: 393-402, 2001 (In French). 
5. Spandidos DA: Oncogenes and tumor suppressor genes as paradigms in oncogenesis. J BUON 12 (Suppl 1): S9-S12, 2007.

6. Shen L, Shi Q and Wang W: Double agents: Genes with both oncogenic and tumor-suppressor functions. Oncogenesis 7: 25, 2018.

7. De Plaen E, Lurquin C, Van Pel A, Mariamé B, Szikora JP, Wölfel T, Sibille C, Chomez P and Boon T: Immunogenic (tum-) variants of mouse tumor P815: Cloning of the gene of tum-antigen P91A and identification of the tum-mutation. Proc Natl Acad USA 85: 2274-2278, 1988.

8. Wirth TC and Kühnel F: Neoantigen targeting-dawn of a new era in cancer immunotherapy? Front Immunol 8: 1848, 2017.

9. Finn OJ: Human tumor antigens yesterday, today, and tomorrow. Cancer Immunol Res 5: 347-354, 2017.

10. Chen DS and Mellman I: Oncology meets immunology: The cancer-immunity cycle. Immunity 39: 1-10, 2013.

11. Jongsma MLM, Guarda G and Spaapen RM: The regulatory network behind MHC class I expression. Mol Immunol 113 16-21, 2019.

12. Shastri N, Nagarajan N, Lind KC and Kanaseki T: Monitoring peptide processing for MHC class I molecules in the endoplasmic reticulum. Curr Opin Immunol 26: 123-127, 2014.

13. Ren Y, Cherukuri Y, Wickland DP, Sarangi V, Tian S, Carter JM, Mansfield AS, Block MS, Sherman ME, Knutson KL, et al: HLA class-I and II restricted neoantigen loads predict overall survival in breast cancer. Oncoimmunology 9: 1744947, 2020.

14. Axelrod ML, Cook RS, Johnson DB and Balko JM: Biological consequences of MHC-II expression by tumor cells in cancer. Clin Cancer Res 25: 2392-2402, 2019.

15. Dantoing E, Piton N, Salaün M, Thiberville L and Guisier F: Anti-PD1/PD-L1 immunotherapy for non-small cell lung cancer with actionable oncogenic driver mutations. Int J Mol Sci 22 : 6288, 2021

16. Kok VC: Current understanding of the mechanisms underlying immune evasion from PD-1/PD-L1 immune checkpoint blockade in head and neck cancer. Front Oncol 10: 268, 2020.

17. Raphael I, Kumar R, McCarl LH, Shoger K, Wang L, Sandlesh P Sneiderman CT, Allen J, Zhai S, Campagna ML, et al: TIGIT and PD-1 immune checkpoint pathways are associated with patient outcome and anti-tumor immunity in glioblastoma. Front Immunol 12: 637146, 2021.

18. Twomey JD and Zhang B: Cancer immunotherapy update: FDA-approved checkpoint inhibitors and companion diagnostics. AAPS J 23: 39, 2021

19. Miller A, Asmann Y, Cattaneo L, Braggio E, Keats J, Auclair D, Lonial S; MMRF CoMMpass Network, Russell SJ and Stewart AK: High somatic mutation and neoantigen burden are correlated with decreased progression-free survival in multiple myeloma. Blood Cancer J 7: e612, 2017.

20. Yi M, Qin S, Zhao W, Yu S, Chu Q and Wu K: The role of neoantigen in immune checkpoint blockade therapy. Exp Hematol Oncol 7: 28, 2018.

21. van den Bulk J, Verdegaal EME, Ruano D, Ijsselsteijn ME, Visser M, van der Breggen R, Duhen T, van der Ploeg M, de Vries NL, Oosting J, et al: Neoantigen-specific immunity in low mutation burden colorectal cancers of the consensus molecular subtype 4. Genome Med 11: 87, 2019.

22. Yarchoan M, Johnson BA III, Lutz ER, Laheru DA and Jaffee EM: Targeting neoantigens to augment antitumour immunity. Nat Rev Cancer 17: 569, 2017.

23. Blass E and Ott PA: Advances in the development of personalized neoantigen-based therapeutic cancer vaccines. Nat Rev Clin Oncol 18: 215-229, 2021.

24. Pardi N, Hogan MJ, Porter FW and Weissman D: mRNA vaccines-a new era in vaccinology. Nat Rev Drug Discov 17: 261-279, 2018

25. Parkhurst MR, Robbins PF, Tran E, Prickett TD, Gartner JJ, Jia L, Ivey $\mathrm{G}, \mathrm{Li}$ YF, El-Gamil M, Lalani A, et al: Unique neoantigens arise from somatic mutations in patients with gastrointestinal cancers. Cancer Discov 9: 1022-1035, 2019.

26. Klebanoff CA and Wolchok JD: Shared cancer neoantigens: Making private matters public. J Exp Med 215: 5-7, 2018.

27. Garcia-Garijo A, Fajardo CA and Gros A: Determinants for neoantigen identification. Front Immunol 10: 1392, 2019.

28. Hutchison S and Pritchard AL: Identifying neoantigens for use in immunotherapy. Mamm Genome 29: 714-730, 2018.

29. Chen F, Zou Z, Du J, Su S, Shao J, Meng F, Yang J, Xu Q, Ding N, Yang Y, et al: Neoantigen identification strategies enable personalized immunotherapy in refractory solid tumors. J Clin Invest 129: 2056-2070, 2019.
30. Hao Q, Wei P, Shu Y,Zhang YG, Xu H and Zhao JN: Improvement of neoantigen identification through convolution neural network. Front Immunol 12: 682103, 2021

31. Bulik-Sullivan B, Busby J, Palmer CD, Davis MJ, Murphy T, Clark A, Busby M, Duke F, Yang A, Young L, et al: Deep learning using tumor HLA peptide mass spectrometry datasets improves neoantigen identification. Nat Biotechnol: Dec 17, 2018 (Epub ahead of print)

32. Guo C, Manjili MH, Subjeck JR, Sarkar D, Fisher PB and Wang XY: Therapeutic cancer vaccines: Past, present, and future. Adv Cancer Res 119: 421-475, 2013

33. Schindler T, Bornmann W, Pellicena P, Miller WT, Clarkson B and Kuriyan J: Structural mechanism for STI-571 inhibition of abelson tyrosine kinase. Science 289: 1938-1942, 2000.

34. Fuster LM and Sandler AB: Select clinical trials of erlotinib (OSI-774) in non-small-cell lung cancer with emphasis on phase III outcomes. Clin Lung Cancer 6 (Suppl 1): S24-S29, 2004.

35. Rosenberg SA, Yang JC and Restifo NP: Cancer immunotherapy: Moving beyond current vaccines. Nat Med 10: 909-915, 2004.

36. de Gruijl TD, van den Eertwegh AJ, Pinedo HM and Scheper RJ: Whole-cell cancer vaccination: From autologous to allogeneic tumor- and dendritic cell-based vaccines. Cancer Immunol Immunother 57: 1569-1577, 2008.

37. Chiang CL, Hagemann AR, Leskowitz R, Mick R, Garrabrant T, Czerniecki BJ, Kandalaft LE, Powell DJ Jr and Coukos G: Day-4 myeloid dendritic cells pulsed with whole tumor lysate are highly immunogenic and elicit potent anti-tumor responses. PLoS One 6: e28732, 2011

38. Bencherif SA, Warren Sands R, Ali OA, Li WA, Lewin SA, Braschler TM, Shih TY, Verbeke CS, Bhatta D, Dranoff G and Mooney DJ: Injectable cryogel-based whole-cell cancer vaccines. Nat Commun 6: 7556, 2015.

39. Lim YT: Vaccine adjuvant materials for cancer immunotherapy and control of infectious disease. Clin Exp Vaccine Res 4: 54-58, 2015.

40. Zhu G, Zhang F, Ni Q, Niu G and Chen X: Efficient nanovaccine delivery in cancer immunotherapy. ACS Nano 11: 2387-2392, 2017.

41. Obeid J, Hu Y and Slingluff CL Jr: Vaccines, adjuvants, and dendritic cell activators-current status and future challenges. Semin Oncol 42: 549-561, 2015.

42. Luo M, Samandi LZ, Wang Z, Chen ZJ and Gao J: Synthetic nanovaccines for immunotherapy. J Control Release 263: 200-210, 2017.

43. Izumoto S: Peptide vaccine. Adv Exp Med Biol 746: 166-177, 2012.

44. Lynn GM, Sedlik C, Baharom F, Zhu Y, Ramirez-Valdez RA, Coble VL, Tobin K, Nichols SR, Itzkowitz Y, Zaidi N, et al: Peptide-TLR-7/8a conjugate vaccines chemically programmed for nanoparticle self-assembly enhance CD8 T-cell immunity to tumor antigens. Nat Biotechnol 38: 320-332, 2020.

45. Ni Q, Zhang F, Liu Y, Wang Z, Yu G, Liang B, Niu G, Su T, Zhu G, Lu G, et al: A bi-adjuvant nanovaccine that potentiates immunogenicity of neoantigen for combination immunotherapy of colorectal cancer. Sci Adv 6: eaaw6071, 2020.

46. Esposito A, Criscitiello C and Curigliano G: Immune checkpoint inhibitors with radiotherapy and locoregional treatment: Synergism and potential clinical implications. Curr Opin Oncol 27: 445-451, 2015.

47. Ott PA, Hu-Lieskovan S, Chmielowski B, Govindan R, Naing A, Bhardwaj N, Margolin K, Awad MM, Hellmann MD, Lin JJ, et al: A Phase Ib trial of personalized neoantigen therapy plus anti-PD-1 in patients with advanced melanoma, non-small cell lung cancer, or bladder cancer. Cell 183: 347-362.e24, 2020.

48. Shaw SM, Middleton J, Wigglesworth K, Charlemagne A, Schulz O, Glossop MS, Whalen GF, Old R, Westby M, Pickford C, et al: AGI-134: A fully synthetic $\alpha$-Gal glycolipid that converts tumors into in situ autologous vaccines, induces anti-tumor immunity and is synergistic with an anti-PD-1 antibody in mouse melanoma models. Cancer Cell Int 19: 346, 2019.

49. Fennemann FL, de Vries IJM, Figdor CG and Verdoes M: Attacking tumors from all sides: Personalized multiplex vaccines to tackle intratumor heterogeneity. Front Immunol 10: 824, 2019.

50. Ott PA, Hu Z, Keskin DB, Shukla SA, Sun J, Bozym DJ, Zhang W, Luoma A, Giobbie-Hurder A, Peter L, et al: An immunogenic personal neoantigen vaccine for patients with melanoma. Nature 547: 217-221, 2017.

51. Keskin DB, Anandappa AJ, Sun J, Tirosh I, Mathewson ND, Li S, Oliveira G, Giobbie-Hurder A, Felt K, Gjini E, et al: Neoantigen vaccine generates intratumoral $\mathrm{T}$ cell responses in phase $\mathrm{Ib}$ glioblastoma trial. Nature 565: 234-239, 2019. 
52. Zeng Y, Zhang W, Li Z, Zheng Y, Wang Y, Chen G, Qiu L, Ke K, Su X, Cai Z, et al: Personalized neoantigen-based immunotherapy for advanced collecting duct carcinoma: Case report. J Immunother Cancer 8: e000217, 2020.

53. Caron E, Aebersold R, Banaei-Esfahani A, Chong $\mathrm{C}$ and Bassani-Sternberg M: A case for a human immuno-peptidome project consortium. Immunity 47: 203-208, 2017.

54. Hellmann MD and Snyder A: Making it personal: Neoantigen vaccines in metastatic melanoma. Immunity 47: 221-223, 2017.

55. Fu C, Zhou L, Mi QS and Jiang A: DC-based vaccines for cancer immunotherapy. Vaccines (Basel) 8: 706, 2020.

56. Wculek SK, Cueto FJ, Mujal AM, Melero I, Krummel MF and Sancho D: Dendritic cells in cancer immunology and immunotherapy. Nat Rev Immunol 20: 7-24, 2020.

57. Zhang R, Yuan F, Shu Y, Tian Y, Zhou B, Yi L, Zhang X, Ding $\mathrm{Z}, \mathrm{Xu} \mathrm{H}$ and Yang L: Personalized neoantigen-pulsed dendritic cell vaccines show superior immunogenicity to neoantigen-adjuvant vaccines in mouse tumor models. Cancer Immunol Immunother 69: 135-145, 2020.

58. Tang L, Zhang R, Zhang $X$ and Yang L: Personalized neoantigen-Pulsed DC vaccines: Advances in clinical applications. Front Oncol 11: 701777, 2021.

59. Carreno BM, Magrini V, Becker-Hapak M, Kaabinejadian S, Hundal J, Petti AA, Ly A, Lie WR, Hildebrand WH, Mardis ER and Linette GP: Cancer immunotherapy. A dendritic cell vaccine increases the breadth and diversity of melanoma neoantigen-specific T cells. Science 348: 803-808, 2015.

60. Charles J, Chaperot L, Hannani D, Bruder Costa J, Templier I, Trabelsi S, Gil H, Moisan A, Persoons V, Hegelhofer H, et al: An innovative plasmacytoid dendritic cell line-based cancer vaccine primes and expands antitumor T-cells in melanoma patients in a first-in-human trial. Oncoimmunology 9: 1738812, 2020.

61. Pastor F, Berraondo P, Etxeberria I, Frederick J, Sahin U, Gilboa E and Melero I: An RNA toolbox for cancer immunotherapy. Nat Rev Drug Discov 17: 751-767, 2018.

62. Lopes A, Vandermeulen G and Préat V: Cancer DNA vaccines: Current preclinical and clinical developments and future perspectives. J Exp Clin Cancer Res 38: 146, 2019.

63. Sahin U, Derhovanessian E, Miller M, Kloke BP, Simon P, Löwer M, Bukur V, Tadmor AD, Luxemburger U, Schrörs B, et al: Personalized RNA mutanome vaccines mobilize poly-specific therapeutic immunity against cancer. Nature 547: 222-226, 2017.

64. Cafri G, Gartner JJ, Zaks T, Hopson K, Levin N, Paria BC, Parkhurst MR, Yossef R, Lowery FJ, Jafferji MS, et al: mRNA vaccine-induced neoantigen-specific $\mathrm{T}$ cell immunity in patients with gastrointestinal cancer. J Clin Invest 130: 5976-5988, 2020.

65. Platten M, Bunse L, Wick A, Bunse T, Le Cornet L, Harting I, Sahm F, Sanghvi K, Tan CL, Poschke I, et al: A vaccine targeting mutant IDH1 in newly diagnosed glioma. Nature 592: 463-468,2021.

66. Tondini E, Arakelian T, Oosterhuis K, Camps M, van Duikeren S, Han W, Arens R, Zondag G, van Bergen J and Ossendorp F: A poly-neoantigen DNA vaccine synergizes with PD-1 blockade to induce T cell-mediated tumor control. Oncoimmunology 8 : 1652539, 2019

67. Li Q, Ren J, Liu W, Jiang G and Hu R: CpG oligodeoxynucleotide developed to activate primate immune responses promotes antitumoral effects in combination with a neoantigen-based mRNA cancer vaccine. Drug Des Devel Ther 15: 3953-3963, 2021.

68. Duperret EK, Perales-Puchalt A, Stoltz R, G HH, Mandloi N, Barlow J, Chaudhuri A, Sardesai NY and Weiner DB: A synthetic DNA, multi-neoantigen vaccine drives predominately MHC class I CD $8^{+} \mathrm{T}$-cell responses, impacting tumor challenge. Cancer Immunol Res 7: 174-182, 2019.

69. Wang $\mathrm{Z}$ and $\mathrm{Wu} \mathrm{X}$ : Study and analysis of antitumor resistance mechanism of PD1/PD-L1 immune checkpoint blocker. Cancer Med 9: 8086-8121, 2020

70. Tan CL, Kuchroo JR, Sage PT, Liang D, Francisco LM, Buck J, Thaker YR, Zhang Q, McArdel SL, Juneja VR, et al: PD-1 restraint of regulatory $\mathrm{T}$ cell suppressive activity is critical for immune tolerance. J Exp Med 218: e20182232, 2021.

71. Buchbinder EI and Desai A: CTLA-4 and PD-1 pathways: Similarities, differences, and implications of their inhibition. Am J Clin Oncol 39: 98-106, 2016.

72. Wisdom AJ, Mowery YM, Riedel RF and Kirsch DG: Rationale and emerging strategies for immune checkpoint blockade in soft tissue sarcoma. Cancer 124: 3819-3829, 2018

73. Xu F, Jin T, Zhu Y and Dai C: Immune checkpoint therapy in liver cancer. J Exp Clin Cancer Res 37: 110, 2018.

74. Pianko MJ, Liu Y, Bagchi S and Lesokhin AM: Immune checkpoint blockade for hematologic malignancies: A review. Stem Cell Investig 4: 32, 2017.
75. Kabacaoglu D, Ciecielski KJ, Ruess DA and Algül H: Immune checkpoint inhibition for pancreatic ductal adenocarcinoma: Current limitations and future options. Front Immunol 9: 1878, 2018.

76. Anagnostou V, Smith KN, Forde PM, Niknafs N, Bhattacharya R, White J, Zhang T, Adleff V, Phallen J, Wali N, et al: Evolution of neoantigen landscape during immune checkpoint blockade in non-small cell lung cancer. Cancer Discov 7: 264-276, 2017.

77. HodiFS,O'DaySJ,McDermottDF,WeberRW,SosmanJA,HaanenJB Gonzalez R, Robert C, Schadendorf D, Hassel JC, et al: Improved survival with ipilimumab in patients with metastatic melanoma. $\mathrm{N}$ Engl J Med 363: 711-723, 2010.

78. Isaacsson Velho P and Antonarakis ES: PD-1/PD-L1 pathway inhibitors in advanced prostate cancer. Expert Rev Clin Pharmacol 11: 475-486, 2018.

79. Wang X, Guo G, Guan H, Yu Y, Lu J and Yu J: Challenges and potential of PD-1/PD-L1 checkpoint blockade immunotherapy for glioblastoma. J Exp Clin Cancer Res 38: 87, 2019.

80. Jiang Y, Chen M, Nie H and Yuan Y: PD-1 and PD-L1 in cancer immunotherapy: Clinical implications and future considerations. Hum Vaccin Immunother 15: 1111-1122, 2019.

81. Peng M, Mo Y, Wang Y, Wu P, Zhang Y, Xiong F, Guo C, Wu X, Li Y, Li X, et al: Neoantigen vaccine: An emerging tumor immunotherapy. Mol Cancer 18: 128, 2019.

82. Liu CJ, Schaettler M, Blaha DT, Bowman-Kirigin JA, Kobayashi DK, Livingstone AJ, Bender D, Miller CA, Kranz DM, Johanns TM and Dunn GP: Treatment of an aggressive orthotopic murine glioblastoma model with combination checkpoint blockade and a multivalent neoantigen vaccine. Neuro Oncol 22: 1276-1288, 2020.

83. Duraiswamy J, Kaluza KM, Freeman GJ and Coukos G: Dual blockade of PD-1 and CTLA-4 combined with tumor vaccine effectively restores T-cell rejection function in tumors. Cancer Res 73: 3591-3603, 2013.

84. Rohaan MW, Wilgenhof S and Haanen JBAG: Adoptive cellular therapies: The current landscape. Virchows Arch 474: 449-461, 2019.

85. Rath JA and Arber C: Engineering strategies to enhance TCR-based adoptive T cell therapy. Cells 9: 1485, 2020.

86. Weber J, Atkins M, Hwu P, Radvanyi L, Sznol M and Yee C; Immunotherapy Task Force of the NCI Investigational Drug Steering Committee: White paper on adoptive cell therapy for cancer with tumor-infiltrating lymphocytes: A report of the CTEP subcommittee on adoptive cell therapy. Clin Cancer Res 17: 1664-1673, 2011.

87. Rohaan MW, van den Berg JH, Kvistborg P and Haanen JBAG: Adoptive transfer of tumor-infiltrating lymphocytes in melanoma: A viable treatment option. J Immunother Cancer 6: 102, 2018.

88. van den Berg JH, Heemskerk B, van Rooij N, Gomez-Eerland R, Michels S, van Zon M, de Boer R, Bakker NAM, Jorritsma-Smit A, van Buuren MM, et al: Tumor infiltrating lymphocytes (TIL) therapy in metastatic melanoma: Boosting of neoantigen-specific $\mathrm{T}$ cell reactivity and long-term follow-up. J Immunother Cancer 8: e000848, 2020

89. Zacharakis N, Chinnasamy H, Black M, Xu H, Lu YC, Zheng Z, Pasetto A, Langhan M, Shelton T, Prickett T, et al: Immune recognition of somatic mutations leading to complete durable regression in metastatic breast cancer. Nat Med 24: 724-730, 2018.

90. Tran E, Robbins PF, Lu YC, Prickett TD, Gartner JJ, Jia L, Pasetto A, Zheng Z, Ray S, Groh EM, et al: T-cell transfer therapy targeting mutant KRAS in cancer. N Engl J Med 375: 2255-2262, 2016 .

91. Sun J, Zhang J, Hu H, Qin H, Liao X, Wang F, Zhang W, Yin Q, Su X, He Y, et al: Anti-tumour effect of neo-antigen-reactive T cells induced by RNA mutanome vaccine in mouse lung cancer. J Cancer Res Clin Oncol 147: 3255-3268, 2021.

92. Rosenberg SA and Restifo NP: Adoptive cell transfer as personalized immunotherapy for human cancer. Science 348: 62-68, 2015.

93. Lu YC, Yao X, Crystal JS, Li YF, El-Gamil M, Gross C, Davis L, Dudley ME, Yang JC, Samuels Y, et al: Efficient identification of mutated cancer antigens recognized by $\mathrm{T}$ cells associated with durable tumor regressions. Clin Cancer Res 20: 3401-3410, 2014.

94. Gros A, Parkhurst MR, Tran E, Pasetto A, Robbins PF, Ilyas S, Prickett TD, Gartner JJ, Crystal JS, Roberts IM, et al: Prospective identification of neoantigen-specific lymphocytes in the peripheral blood of melanoma patients. Nat Med 22: 433-438, 2016.

95. Bailey P,Chang DK, Forget MA,Lucas FA, Alvarez HA,HaymakerC, Chattopadhyay C, Kim SH, Ekmekcioglu S, Grimm EA, et al: Exploiting the neoantigen landscape for immunotherapy of pancreatic ductal adenocarcinoma. Sci Rep 6: 35848, 2016.

This work is licensed under a Creative Commons Attribution-NonCommercial-NoDerivatives 4.0 International (CC BY-NC-ND 4.0) License. 